\title{
Myxoid Chondrosarcoma of the Hyoid Bone
}

\author{
Kinjal Majumdar, ${ }^{1}$ Nirmalya Samanta, ${ }^{2}$ Devjani Ghosh Shrestha, ${ }^{3}$ Kasturi Mondal, ${ }^{2}$ Utpal Jana ${ }^{2}$
}

\section{Introduction}

\section{ABSTRACT}

Chondrosarcoma of hyoid bone is very rare with only 19 cases still reported. We therefore, present this case report for the interest of medical literature to make clinicians aware of the disease.

\section{Case Report}

They usually present as a slow growing upper neck mass. Computed tomography (CT) and magnetic resonance imaging (MRI) are useful radiologic investigations. The tumour was resected through a trans-cervical approach. Definite diagnosis was made by postoperative histopathology and immunohistochemistry.

\section{Discussion}

Surgical excision is the treatment of choice for local control. Incomplete removal is a risk factor for recurrence and possible dedifferentiation. Long term follow up is necessary.

Keywords

Chondrosarcoma; Hyoid Bone; Immunohistochemistry

$\mathrm{C}$ hondrosarcoma is the third most common primary bone tumour accounting for $11 \%$ of all primary bone tumours, ${ }^{1}$ after osteosarcoma and multiple myeloma. ${ }^{2}$ Chondrosarcoma commonly occurs in pelvis, femur, humerus and ribs. Only in $1-12 \%$ cases, ${ }^{1}$ it can be seen in head and neck region with skull base, maxilla and larynx being affected.

Chondrosarcoma of the hyoid bone is an extremely rare entity. We searched for published literature before June,2015 in MEDLINE and PubMed using the terms "chondrosarcoma" and "hyoid" which led to only 19 cases still reported. Here, we report a case of myxoid chondrosarcoma of hyoid bone along with its preoperative imaging studies, perioperative findings and postoperative complications, histopathology and immunohistochemistry.

\section{Case report}

A 50 years old gentleman presented to us with a slow growing, painless, firm, globular mass in upper neck in the midline which he first noticed 7 months back. The mass was adherent to underlying structures and moved with deglutition. (Fig.1)
He has a past history surgical excision of haemangioendothelioma of epiglottis three years ago. Neck was found to be clinically negative for lymph nodes. Fibre-optic laryngoscopy revealed normal laryngeal and hypopharyngeal subsites. CT Scan revealed a well demarcated peripherally enhancing hypodense lesion at base of the tongue extending to floor of the mouth involving the body and right greater cornu of the hyoid bone with thin enhancing septae within the lesion. No cervical lymphadenopathy was noted. (Fig.2)

T1 weighted post-contrast study in magnetic resonance angiography (MRA) showed irregular enhancing lesion involving floor of mouth on right side with oedema around it suggestive of vascular lesion with branches of external carotid artery appeared to drain the above areas

1 - Department of ENT, HealthCare Global Enterprises Ltd., Bangalore, Karnataka.

2 - Department of ENT, Nil Ratan Sircar Medical College, Kolkata.

3 - Department of ENT, Medica Superspeciality Hospital, Kolkata.

Corresponding author:

Dr Kinjal Majumdar

email: drkinjalmajumdar@gmail.com 


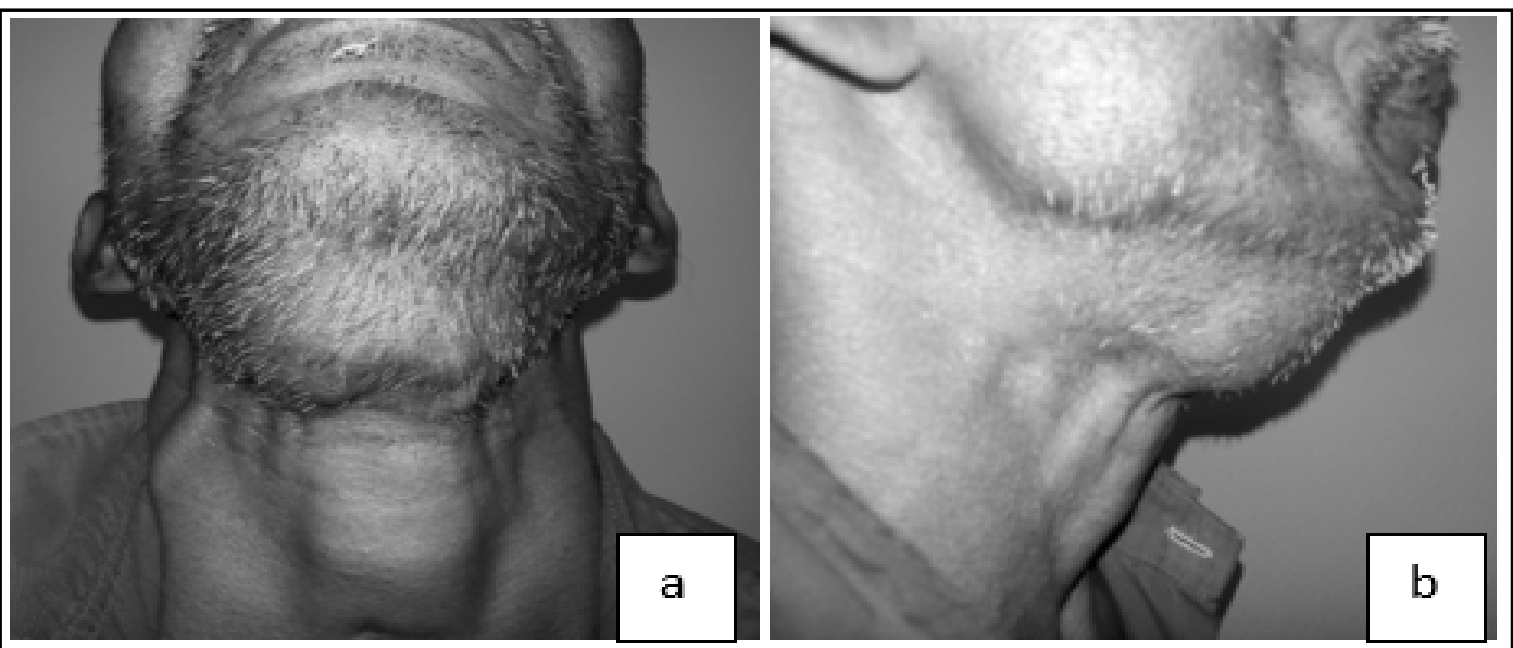

Fig. 1. Clinical presentation. a. Front view and $b$. Side view of the mass, $5 \mathrm{~cm} \times 3 \mathrm{~cm}$, situated in the upper neck in the midline, firm and non-tender on palpation

\section{(Fig. 3)}

Preoperative fine needle aspiration cytology (FNAC) showed atypical cells arranged mostly singly, also in clusters and ill formed papillae. Individual cells were round or oval to spindle with moderate amount of cytoplasm and hyperchromatic nucleus. Many of the cells were histiocytoid or epitheloid in appearance. Stromal fragments were seen in background. Overall cytomorphological picture suggested metastatic deposit of epiglottic haemangioendothelioma with an eye on the past operative history of the patient.

Surgical excision was planned via a trans-cervical route with upper crease neck incision. Tumour was found to be extensively attached to body and right greater cornu of the hyoid bone along with geniohyoid, mylohyoid, sternohyoid and thyrohyoid. Right hypoglossal nerve was found to be severely adhered to the tumour. Tumour dissected out in toto along with the body and right greater cornu of the hyoid bone. Right hypoglossal nerve was sacrificed for complete removal

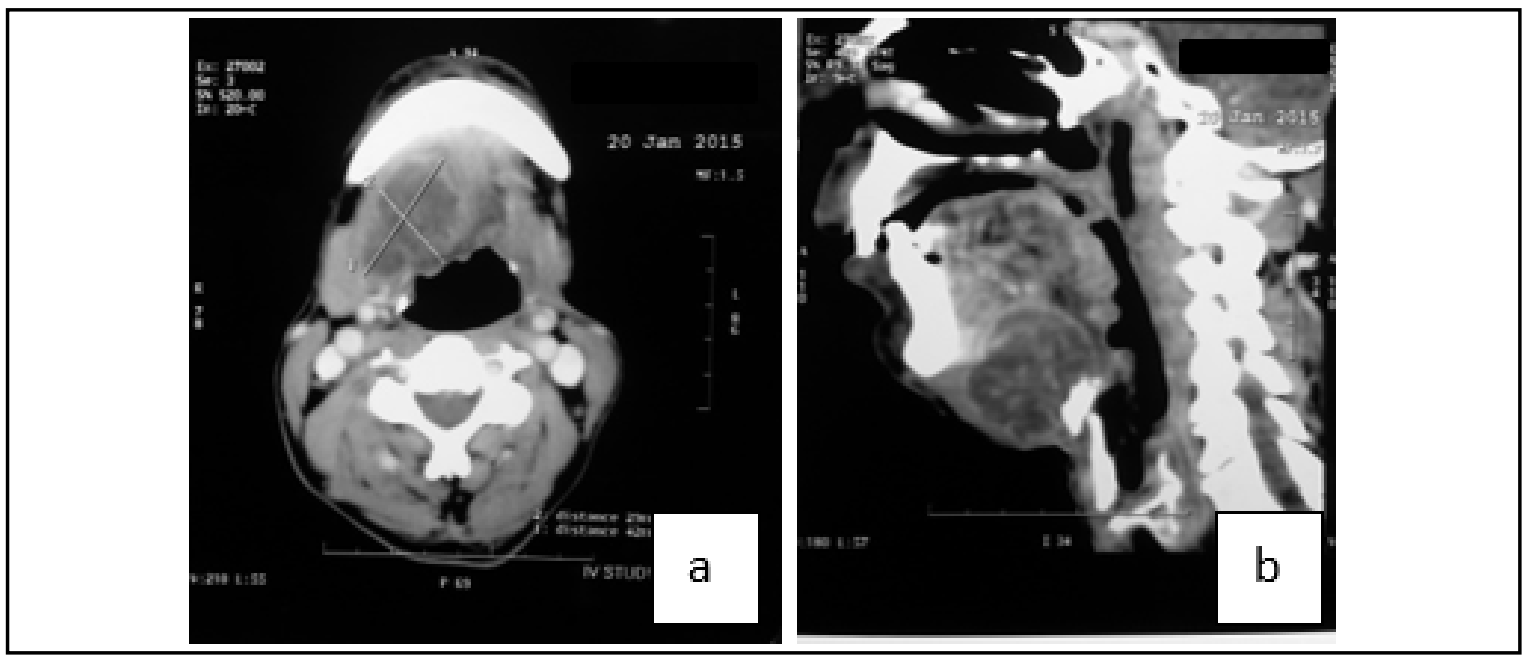

Fig. 2. Contrast enhanced computed tomography Axial cut (a) and Sagittal cut (b) showing a well marginated peripherally enhancing hypodense lesion measuring $(3.6 \times 4.2 \times 3.7) \mathrm{cm}$ with thin enhancing sepatae 


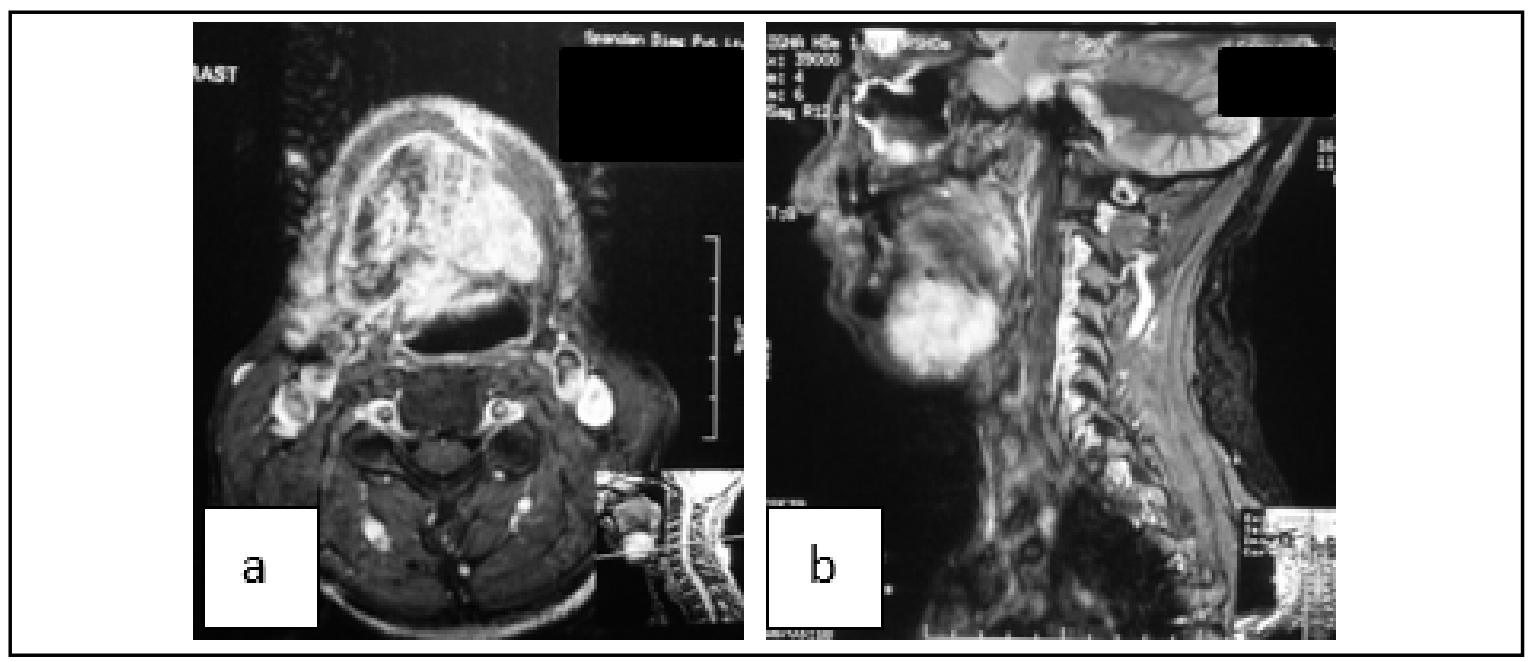

Fig. 3. Magnetic resonance imaging Axial cut (a) and Sagittal cut (b) T1 weighted post contrast study is showing irregular enhancing lesion slightly more on the right side with oedema around it. Lesion measures about $45 \mathrm{~mm}$ in antero-posterior, $13 \mathrm{~mm}$ in lateral and $36 \mathrm{~mm}$ in cranio-caudal dimension.

of the tumour. Pharynx was not entered. (Fig. 4)

Post operatively patient developed right hypoglossal nerve palsy and dysphagia. No additional complication noted in one year follow-up.

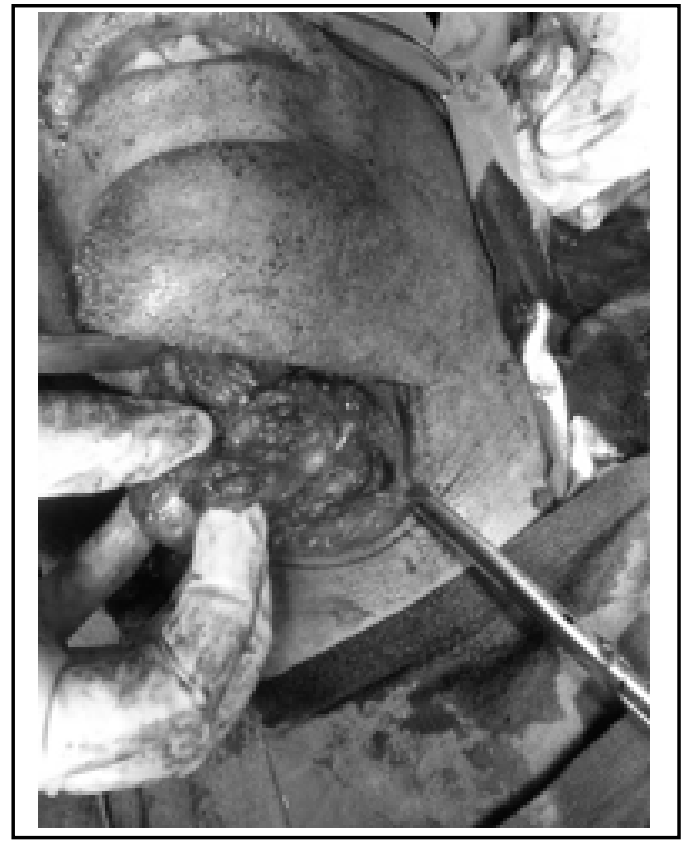

Fig. 4. Surgical excision of the tumour along with body and right greater cornu of the hyoid bone.
Postoperative histopathology by examining Haematoxylin and Eosin, Periodic Acid Schiff and van Gieson stained sections revealed poorly differentiated sarcomatous lesion, presumably chondrosarcoma. Surgical margins were found to be clear of tumour. Subsequent immunohistochemistry showed the tumour to be immunoreactive (score $2+$ in neoplastic cells) to S100 marker suggestive of myxoid chondrosarcoma.

No recurrence noted in one year follow-up.

\section{Discussion}

Chondrosarcoma is the third most common primary bone tumour, pelvis being the most common site of occurrence. Chondrosarcoma arising in head and neck region is rare. A review by National Cancer Database (NCDB) in the United States identified 400 cases of head and neck chondrosarcoma in a ten year period from 1985 to $1995 .{ }^{1}$ But chondrosarcoma of hyoid is very rare with only 19 cases still reported in international literature.

From the NCDB report, the average age of patients with chondrosarcoma of the head and neck region is 51 years, ${ }^{3}$ which very well correlates with our case. There is slight male predominance and ethnicity reveals that non-Hispanic white constitutes more than 
$86 \%$ of cases. ${ }^{4}$ Only a small percentage of cases with regional and distant metastases at diagnosis with 5.6\% and $6.7 \%$, respectively, are found in the NCDB report. ${ }^{5}$ On the whole, it is a low-grade, slow-growing type of sarcoma. It frequently presents as a painless mass originating either within the bone or outside the bone. From this, these tumours are typically classified as osseous and extraosseous ones, which can be graded from one to three based on the histological appearance, ${ }^{6}$ with grade 3 bearing the worst prognosis. In our case, the tumour fell in grade 2 category consistent with myxoid chondrosarcoma. Rarely, a chondrosarcoma will dedifferentiate to a high-grade spindle cell sarcoma with a very bad prognosis. ${ }^{7}$ So a long term follow up of these patients is necessary.

Generally, the most appropriate imaging modality in a case of chondrosarcoma is CT scan to detect bone destruction. Intra-mural calcification may be noted in $75 \%$ of the cases. ${ }^{8,9}$ MRI is useful to detect surrounding soft tissue invasion. Signal intensity is low in T1 weighted image and high in $\mathrm{T} 2$ images, with signal void in calcified areas. A typical mosaic pattern is seen in high intensity T2 images. ${ }^{10}$ In our case, although CT scan revealed no intra-mural calcification, thin enhancing septae were seen within the lesion. MRA gave additional information about possibility of branches of external carotid artery draining the area. Preoperative FNAC, though, did not produce any valuable information.

Treatment is by surgical excision followed by reconstruction, if necessary. Clear margins of excision are absolutely essential for local control. ${ }^{11,12}$ Although consideration of adjuvant radiotherapy may be entertained, particularly in high-grade tumours.

Postoperatively the patient has right hypoglossal nerve palsy and complaint of dysphagia. Histopathological examination revealed a poorly differentiated sarcomatous lesion. Subsequently, immunohistochemistry was carried out using CD31 and S100 markers. CD31 was found to be non-immunoreactive (score 0 in neoplastic cells) ruling out Ewing sarcoma. ${ }^{13}$ Whereas, $26-50 \%$ of neoplastic cells were found to be immunoreactive to $\mathrm{S} 100$ (score $2+$ ), consistent with myxoid chondrosarcoma.

The NCDB report, reveals a surprisingly high survival of head and neck chondrosarcoma at $87.2 \%$ five-year and $70.6 \%$ ten-year survival, with $59.5 \%$ undergoing surgery alone and $21 \%$ having adjuvant radiotherapy.

\section{References}

1. Koch BB, Karnell LH, Hoffman HT et al. National cancer database report on chondrosarcoma of the head and neck. Head Neck 2000; 22; 408-25

2. Manaster BJ. Skeletal radiology: handbooks in radiology, Chicago, Year Book Medical Publishers, 1989

3. Flint PW, Haughey BH, Lund VJ et al. Cummings Otolaryngology-Head and Neck Surgery, 6th edition, Philadelphia, Elsevier Saunders, 2015

4. Batsakis JG, Solomon AR, Rice DH. The pathology of head and neck tumors: neoplasm of cartilage, bone, and the notochord, part 7; Head Neck Surg. 1980; 3; 43-57

5. Lee SY, Lim YC, Song MH, Seok JY, Lee WS, Choi EC. Chondrosarcoma of the Head and Neck; Yonsei Med J. 2005; 46; 2 : 228-32

6. Dr Frank Gaillard. Chondrosarcoma grading; URL: radiopaedia. org/articles/chondrosarcoma-grading

7. Staals EL, Bacchini P, Bertoni F. Dedifferentiated central chondrosarcoma; Cancer 2006; 106; 2682-91

8. Somer F, Perdieus D, Van Den Hauwe L, Lemmens L, Schillebeeckx J. Chondrosarcoma of the hyoid bone. Eur Radiol. 2000; 10; 2; 308-9

9. Hediger R, McEniff N, Karmody C, Eustace S. Recurrent chondrosarcoma of the hyoid bone. Clin Imaging 1997; 21: 1; 6972

10. Itoh K, Nobori $\mathrm{T}$, Fukuda $\mathrm{K}$, Furuta $\mathrm{S}$, Ohyama $\mathrm{M}$. Chondrosarcoma of the hyoid bone. J Laryngol Otol. 1993; 107(7): 6426

11. Fiorenza F, Abudu A, Grimer RJ, Carter SR, Tillman RM, Ayoub K et al. Risk factors for survival and local control in chondrosarcoma of bone. J Bone Joint Surg Br. 2002; 84(1): 93-9

12. Lee FY, Mankin HJ, Fondren G, Gebhardt MC, Springfield DS, Rosenberg AE et al. Chondrosarcoma of bone: an assessment of outcome; J Bone Joint Surg Am. 1999; 81(3): 326-38

13. Teicher BA, Bagley RG, Rouleau C, Kruger A, Ren Y, Kurtzberg L. Characteristics of human Ewing/PNET sarcoma models. Ann Saudi Med. 2011; 31; 2; 174-82. 